\title{
A formação, o trabalho e a identidade profissional do Professor de Computação: um mapeamento sobre a Licenciatura em Computação
}

\author{
Fernando Paiva ${ }^{1}$, Pietro Bompet ${ }^{2}$, Emilayne Corlett ${ }^{3}$, \\ Ecivaldo Matos ${ }^{4}$, Anna Friedericka Schwarzelmüller ${ }^{5}$
}

Departamento de Ciência da Computação - Universidade Federal da Bahia (UFBA) Grupo de Pesquisa e Extensão em Informática, Educação e Sociedade - Onda Digital Av. Adhemar de Barros, s/n - Ondina - CEP 40.170-110 - Salvador - BA - Brasil

\{luizfb, pietro.bompet, emilayne.corlett, ecivaldo, frieda\}@ufba.br

\begin{abstract}
It is necessary to build a holistic and critical view about the formation of the professional responsible for establishing relationships between Computing and Basic Education. This article presents the available evidence in the relevant electronic bases in the area of Computing and Education that approach on the teacher training of the licentiate in Computing in Brazil from a Systematic Mapping of the literature that identified research on the challenges and the difficulties of this professional. The results point to a absence of research about of the Computing Professor.

Resumo. É necessário construir visão holística e crítica a respeito da formação do profissional responsável por estabelecer relações entre a Computação e a Educação Básica. Este artigo apresenta as evidências disponíveis nas bases eletrônicas relevantes na área de Computação $e$ Educação que abordam sobre a formação docente do licenciado em Computação no Brasil a partir de um Mapeamento da literatura que identificou pesquisas sobre os desafios e as dificuldades desse profissional. Os resultados apontam para uma escassez de pesquisas sobre o Professor de Computação.
\end{abstract}

\footnotetext{
${ }^{1}$ Licenciado em Ciência da Computação pela Universidade Federal da Paraíba (UFPB). Mestrando em Ciência da Computação do Programa de Pós-graduação em Ciência da Computação (PGCOMP) da Universidade Federal da Bahia (UFBA).

${ }^{2}$ Licenciando em Computação pela Universidade Federal da Bahia (UFBA). Presidente do Diretório Acadêmico do curso de Licenciatura em Computação da UFBA.

${ }^{3}$ Licenciada em Ciência da Computação pela Universidade Federal da Paraíba (UFPB). Mestranda em Ciência da Computação do Programa de Pós-graduação em Ciência da Computação (PGCOMP) da Universidade Federal da Bahia (UFBA).

${ }^{4}$ Professor Adjunto do Departamento de Ciência da Computação (DCC) e do Programa de Pós-graduação em Ciência da Computação (PGCOMP) da Universidade Federal da Bahia (UFBA). Doutor em Educação pela Universidade de São Paulo (USP). Coordenador do curso de Licenciatura em Computação da UFBA e líder do Grupo de Pesquisa e Extensão em Informática, Educação e Sociedade - Onda Digital.

${ }^{5}$ Mestre em Ciência da Informação pela Universidade Federal da Bahia (UFBA). Professora Adjunta do Departamento de Ciência da Computação (DCC) da UFBA. Pesquisadora do Grupo de Pesquisa e Extensão em Informática, Educação e Sociedade - Onda Digital.
} 
VI Congresso Brasileiro de Informática na Educação (CBIE 2017)

Anais dos Workshops do VI Congresso Brasileiro de Informática na Educação (WCBIE 2017)

\section{Introdução}

A Computação na Educação Básica é vista como imprescindível e sua contribuição para o processo educativo proporciona uma formação crítica e reflexiva pertinentes às exigências e necessidades de nossa realidade. Para que essa contribuição de fato ocorra, Matos (2017, p. 49) explica que é preciso que os cientistas e educadores reconheçam a necessidade de articulação política-pedagógica com as outras áreas de estudo e com a Escola. Pois a "educação básica brasileira irá requerer a formação de professores especializados para atuarem nas escolas” apontam França e Tedesco (2015, p. 1467).

No entanto, as Diretrizes Curriculares Nacionais da Educação Básica (DCN-EB) não contemplam a Computação como um componente curricular, principal campo de atuação do professor de Computação, egresso da Licenciatura em Computação. Apesar disso, desde meados da década de 1990, oriundo de um trabalho iniciado nos idos anos 1960, os computadores fazem parte do ambiente escolar como instrumentos de/para aprendizagem. Contudo, atualmente sabe-se que "lançar computadores na sala de aula não é a maneira mais eficaz de ensinar Computação", como evidencia Wing e Stanzione (2016, p. 2). Compete ao professor de Computação o trabalho de ensinar Computação aos escolares para além das máquinas. Esse, talvez, seja um dos principais desafios a ser superado em sua carreira.

Dentre as competências estabelecidas pelas Diretrizes Curriculares Nacionais para a Formação de Professores da Educação Básica ${ }^{6}$, o Professor de Computação deve ser um sujeito preparado para oferecer o ensino visando a aprendizagem do aluno pautada no uso de tecnologias contemporâneas e de metodologias, estratégias e materiais de apoio inovadores.

Considerando as necessidades apontadas por essas diretrizes, o processo de formação do licenciando em Computação tem o objetivo de formar profissionais que além de ensinar Computação sejam capazes de desenvolver e usar tecnologias compreendidas nesta pesquisa a partir da visão de Pinto (2005) - como o conjunto de todas as técnicas e habilidades do ato produtivo do ser humano - que auxiliem tanto na Educação Básica, Profissional e Técnica, como em outros campos e níveis de Educação.

Nesse processo de articulação política-pedagógica, o Licenciado em Computação é um profissional que compreende quais elementos, conceitos, fundamentos e raciocínios computacionais fazem parte dessa articulação. Logo surge a seguinte pergunta: Quem é o profissional Licenciado em Computação? Quem é o Professor de Computação? Além dessas indagações, Matos (2013) aponta a necessidade de uma "reflexão mais profunda sobre esse profissional que tem concluído o curso superior com pouco reconhecimento pela área de Computação, tampouco pela Educação" (p. 29). E nesse mesmo trabalho, o autor deixa evidente a "necessidade de definição e reconhecimento de uma identidade profissional própria do licenciado enquanto educador" (p. 27). As respostas para essas perguntas exigem visão holística e crítica a respeito da formação, do trabalho e da identidade do Professor de Computação.

Entretanto, o Brasil possui o grande desafio de obter profissionais qualificados para ensinar Computação, como apontado por Souza, Morais e Júnior (2015). Essa falta

${ }^{6}$ Resolução CNE/CES 5/2016. Diário Oficial da União, Brasília, 17 de nov de 2016, Seção 1, págs. 22-24. 
de professores para ensinar Computação na Educação Básica também é um desafio apontado por Wing e Stanzione (2016) que vêem essa lacuna como oportunidade de pesquisa. Araújo, Andrade e Guerrero (2016) também destacam os desafios na formação de professores para o ensino de Computação para escolares. Com o objetivo de conhecer melhor quem é o profissional - Professor de Computação - formado a partir da Licenciatura em Computação (LC), este artigo retrata resultados preliminares de um estudo exploratório acerca das pesquisas sobre o tema.

\section{Procedimentos metodológicos}

Este mapeamento da literatura apresenta evidências disponíveis sobre a formação docente do professor de Computação em pesquisas realizadas a partir de 1997 (ano de criação do primeiro curso de licenciatura em Computação no Brasil) até 2016. O objetivo principal foi quantificar e identificar trabalhos a fim indicar desafios e dificuldades que fazem parte do processo de formação do professor de Computação. Com a seguinte questão norteadora: Quais são os desafios e as dificuldades de formação do professor de Computação?

Utilizou-se uma String de busca simples, com as palavras-chave ("licencia" and "professor de computação" or "professor de informática"). Optou-se usar o termo 'licencia' por retornar todas as palavras-chave derivadas desse radical, como: "licenciando em computação", "licenciatura em computação", "licenciado em computação", "licenciatura em informática", "licenciando em informática" e "licenciado em informática".

As Bases de Dados utilizadas foram eventos e um periódico da Comunidade Informática na Educação no Brasil, são eles: Simpósio Brasileiro de Informática na Educação $(\mathrm{SBIE})^{7}$, Congresso Brasileiro de Informática na Educação $(\mathrm{CBIE})^{8}$, Workshop de Informática na Educação (WIE) ${ }^{9}$, Workshop sobre Educação e Computação (WEI) ${ }^{10}$ e a Revista Brasileira de Informática na Educação (RBIE) ${ }^{11}$. Com a utilização da ferramenta Mendeley®, todos os trabalhos desses eventos e periódico formaram um banco de dados.

\subsection{Estratégia e processo de busca}

O banco de dados foi separado por pastas de cada evento e periódico (Figura 1: retângulo 1) e os trabalhos armazenados em subpastas organizadas pelo ano de sua publicação. As palavras-chave que compõem a String de busca foram aplicadas separadamente dentro de cada pasta de evento e periódico da seguinte forma: dentro do banco de dados criado na ferramenta Mendeley® foi selecionada a pasta desejada para aplicar a palavra-chave; na caixa de busca (Figura 1: retângulo 4) foi escrito o primeiro termo "licencia"; os artigos obtidos nessa busca foram armazenados em outra pasta intitulada 'Licenciatura em Computação (Resultados)' - contendo subpastas com os nomes das bases de dados pesquisadas (Figura 1: retângulo 2); em seguida a caixa de

\footnotetext{
${ }^{7}$ SBIE:http://www.br-ie.org/pub/index.php/sbie/index

${ }^{8}$ CBIE: http://br-ie.org/pub/index.php/wcbie

${ }^{9}$ WIE: http://www.br-ie.org/pub/index.php/wie

${ }^{10}$ WEI: http://www.lbd.dcc.ufmg.br/bdbcomp/servlet/PesquisaEvento?evento=WEI

${ }^{11}$ RBIE: http://www.br-ie.org/pub/index.php/rbie
} 
VI Congresso Brasileiro de Informática na Educação (CBIE 2017)

Anais dos Workshops do VI Congresso Brasileiro de Informática na Educação (WCBIE 2017)

busca foi limpa e o procedimento repetido para a palavra-chave "professor de computação" e logo depois para "professor de informática". A soma dos dados obtidos nas três buscas (Figura 1: retângulo 3) de cada evento ou periódico é a quantidade de artigos encontrados no evento ou periódico pesquisado.

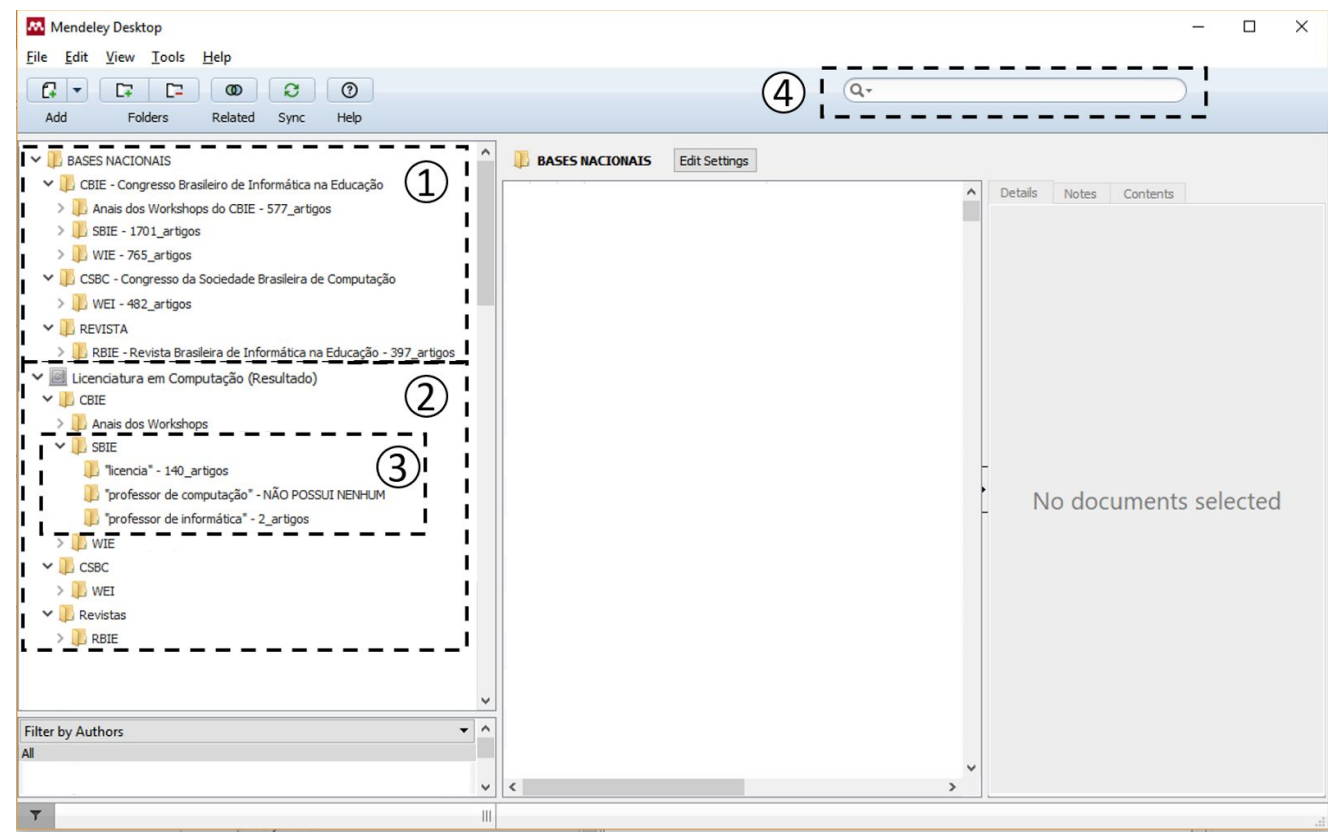

Figura 1. Tela do Mendeley®.

\subsection{Critérios de inclusão e exclusão}

Foram estabelecidos quatro critérios de inclusão e cinco de exclusão. Para a inclusão de um trabalho na pesquisa foi realizada uma análise do título, palavras-chave, resumo e leitura dinâmica do texto completo, após o processo de inclusão os artigos foram classificados por sua relevância em relação à questão de investigação.

Os critérios de inclusão(CI) utilizados foram:

- CI-1 - arquivos disponíveis para download;

- CI-2 - incluir artigos que tratem sobre o processo de formação docente do licenciado em Computação;

- CI-3 - incluir artigos que apresentam os desafios e as dificuldades da formação na licenciatura em Computação;

- CI-4 - incluir artigos que apresentam práticas de licenciados e licenciandos em Computação na Educação Básica.

Durante o processo de análise dos trabalhos também foram aplicados os seguintes critérios de exclusão (CE):

- CE-1 - excluir artigos que não estiverem completos [Não considerar Artigos Curtos (Short Papers), apenas Completos (Full Papers)];

- CE-2 - excluir artigos duplicados; 
VI Congresso Brasileiro de Informática na Educação (CBIE 2017)

Anais dos Workshops do VI Congresso Brasileiro de Informática na Educação (WCBIE 2017)

- CE-3 - excluir artigos que não tratem sobre os desafios e as dificuldades da formação do professor de Computação;

- CE-4 - excluir artigos que não estejam na Língua Portuguesa;

- CE-5 - excluir artigos não disponíveis para download.

Para a aplicação desses critérios foi criada uma planilha Google ${ }^{\circledR}$ para concentrar os dados: Título - Autores - Ano - Instituição - Estado - Evento e Periódico Palavra-chave - Critérios de Inclusão e Exclusão - Relevância; e auxiliar na visualização e organização dessas informações para realização da análise.

\section{Resultados e análise dos dados}

Esta seção apresenta alguns resultados encontrados no Mapeamento, bem como a análise dos dados e em seguida, respostas para questão norteadora.

\subsection{Informações gerais sobre os estudos primários}

As bases de dados pesquisadas retornaram 3.922 (três mil novecentos e vinte e dois) trabalhos que foram organizados em um banco de dados na ferramenta Mendeley®. Depois da aplicação da String de busca no banco de dados, foram retornados 586 (quinhentos e oitenta e seis) trabalhos primários que tratam sobre professor de Computação. Os artigos dessa busca foram organizados em uma planilha, em seguida, uma melhor análise dos estudos primários foi realizada, identificando como relevantes apenas àqueles que se enquadraram nos critérios de inclusão e exclusão expostos na seção 2.2, permanecendo na pesquisa 50 (cinquenta) $\operatorname{artigos}^{12}$ científicos (Tabela 1).

Tabela 1. Quantidade de publicações por conferência/revista

\begin{tabular}{|c|l|c|c|c|c|}
\hline \multicolumn{2}{|c|}{$\begin{array}{c}\text { Bases de Dados } \\
\text { Pesquisadas }\end{array}$} & $\begin{array}{c}\text { Banco de } \\
\text { Dados }\end{array}$ & $\begin{array}{c}\text { Aplicação } \\
\text { da String }\end{array}$ & Critérios de Exclusão/Inclusão \\
\cline { 5 - 6 } & & Excluídos & Incluídos \\
\hline CSBC & Anais do WEI & 482 & 93 & 73 & 20 \\
\hline \multirow{3}{*}{ CBIE } & Anais do WIE & 765 & 186 & 175 & 11 \\
\cline { 2 - 6 } & Anais do SBIE & 1701 & 142 & 134 & 8 \\
\cline { 2 - 6 } & Anais do CBIE & 577 & 104 & 96 & 8 \\
\hline Revista & RBIE & 397 & 61 & 58 & 3 \\
\hline \multicolumn{2}{|c|}{ TOTAL } & $\mathbf{3 9 2 2}$ & $\mathbf{5 8 6}$ & $\mathbf{5 3 6}$ & $\mathbf{5 0}$ \\
\hline
\end{tabular}

\footnotetext{
${ }^{12}$ Referências dos artigos selecionados no mapeamento: https://goo.gl/uvYzfR
} 
VI Congresso Brasileiro de Informática na Educação (CBIE 2017)

Anais dos Workshops do VI Congresso Brasileiro de Informática na Educação (WCBIE 2017)

Ao analisar as fontes de busca separadamente, podemos notar que os Anais do WIE e do SBIE são as bases de dados que possuem o maior número de artigos publicados relacionados com a String. Embora o WIE apresente expressiva quantidade de trabalhos retornados, ao final da seleção apenas 11 artigos foram incluídos. A RBIE foi a fonte que retornou menos artigos, quando comparado às demais bases utilizadas, com apenas 3 artigos incluídos.

\subsection{Alguns resultados dos estudos primários}

Apesar da primeira Licenciatura em Computação no Brasil datar do ano de 1997, o primeiro trabalho encontrado sobre a formação docente do professor de Computação data de 2004, quando começou-se a produzir trabalhos (indexados nessas bases) sobre o tema (Figura 2).

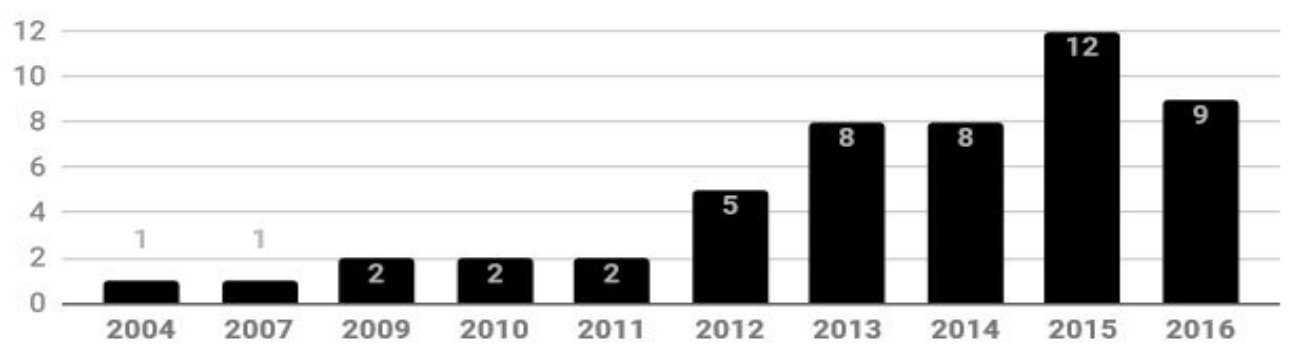

Figura 2. Quantidade de artigos por ano

Conforme Figura 3, o estado que mais publica artigos sobre formação docente do professor de Computação é a Paraíba, seguido de Pernambuco. A Região Nordeste responde por 54\% dessas publicações (27 artigos); sendo, portanto, a região que mais publica. Um dos possíveis motivos para o estado da Paraíba ser o mais representativo em publicações é a quantidade de cursos de LC disponíveis no estado, atualmente possuindo três cursos.



Figura 3. Quantidade de artigos por estado

Conforme a Figura 4, a maioria dos artigos foram escritos por pesquisadores da Universidade Federal da Paraíba (UFPB), com nove artigos ao total. Em seguida, a Universidade de Pernambuco (UPE) com sete artigos. Essa maior representatividade da UFPB pode ser devido à existência de dois cursos de LCC - UFPB/Campus IV e UFPB/Virtual. 
VI Congresso Brasileiro de Informática na Educação (CBIE 2017)

Anais dos Workshops do VI Congresso Brasileiro de Informática na Educação (WCBIE 2017)

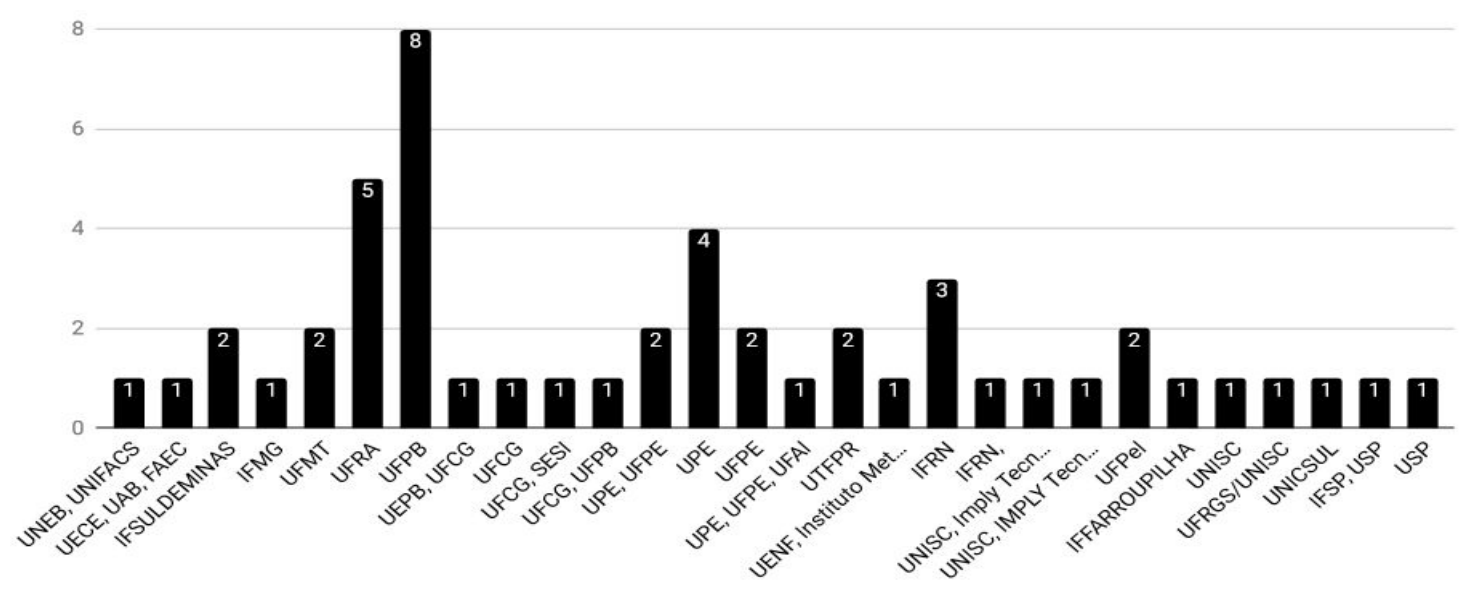

Figura 4. Quantidade de artigos publicados por instituição de Ensino Superior

Os termos "Professor de Computação" e "Professor de Informática" apareceram em apenas nove dos 50 artigos que tratam sobre o profissional que ensina Computação (cf. Figura 5).

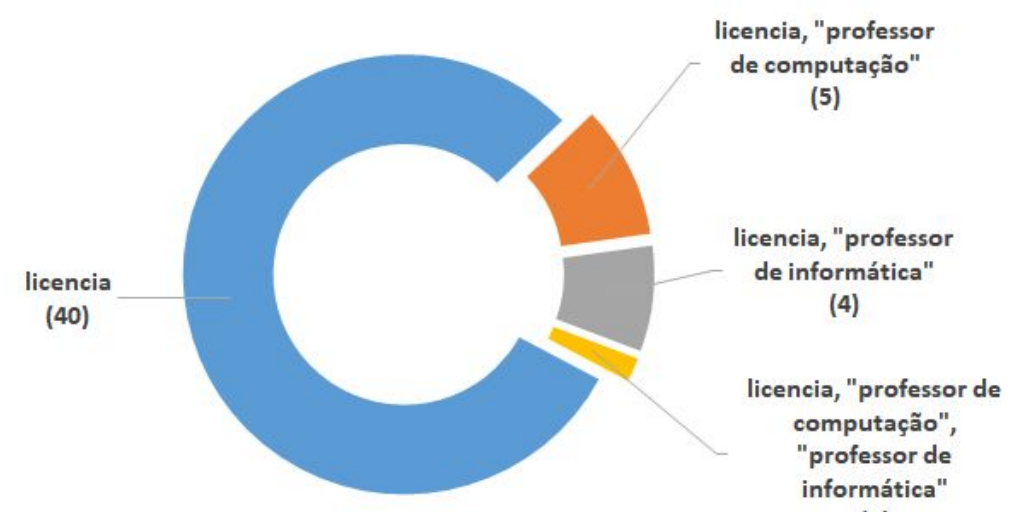

(1)

Figura 5. Quantidade de artigos retornados por palavra chave da String de busca

\subsection{Resposta à questão norteadora}

Nos artigos identificados por meio desse mapeamento diversos autores apresentam desafios e práticas dos professores de Computação, tratadas como oportunidades para esses profissionais. Wing e Stanzione (2016) ressaltam que o principal desafio prático é que não temos professores de Computação para ensinar na Educação Básica. Os autores apontam essa falta como um espaço de oportunidade. E os resultados desse mapeamento evidenciam para a escassez da atuação desse profissional atuante na Educação Básica.

Matos e Silva (2012) explicam que a formação do profissional por meio do curso de Licenciatura em Computação está além dos conhecimentos de natureza científica e tecnológica, envolve também uma terceira dimensão formativa: o conhecimento pedagógico. O desenvolvimento do conhecimento pedagógico é um desafio que faz parte dos saberes profissionais na formação do licenciando em Computação.

Conforme apresentado por Souza, Morais e Júnior (2015), quando o curso de 
Licenciatura em Computação é posto sob análise, os fatores determinantes para o crescimento da evasão dos alunos são "o desprestígio da profissão, os baixos salários e as más condições de trabalho" (ibid. p. 01).

O egresso do curso de Licenciatura em Computação é, a priori, um profissional que possui formação didático-pedagógica capaz de exercer a docência em todas as modalidades da Educação Básica na área da Computação [Sociedade Brasileira de Computação - SBC 2017]. Deve-se compreender que esse profissional vai além disso e possui a capacidade de desenvolver várias competências e habilidades computacionais, estabelecendo relações entre o ensino e aplicação das teorias e recursos computacionais dentro do ambiente escolar, conforme Referenciais de Formação da SBC (2017) ${ }^{13}$.

\section{Considerações Finais}

A maioria dos artigos advindos deste mapeamento evidenciam que a função do professor de Computação, era apenas ensinar a usar tecnologias digitais, mas percebeu-se que nos últimos anos há possibilidade de ensinar a Computação para além das máquinas, a partir dos Raciocínios Computacionais. Mas os resultados não apresentam trabalhos sobre os processos de ensino e didática Computacional.

Outro ponto abordado é a falta de participação do professor de Computação em processos de criação, mudanças e políticas curriculares. Além de constatada a falta de identidade desse profissional, visto que quando os artigos referem-se ao egresso da Licenciatura em Computação a terminologia utilizada não é "professor de Computação". Também são poucos os trabalhos que aprofundam de forma teórica o campo de atuação deste profissional, estabelecendo a sua identidade.

No mapeamento em apenas 5 relatos de experiências são apresentadas práticas e exemplos de ensino de Computação a partir de políticas de inclusão do licenciando de Computação na Educação Básica, como o Programa Institucional de Bolsas de Iniciação à Docência (PIBID), mostrando a contribuição dessas políticas na formação inicial desse docente.

O objetivo deste Mapeamento foi quantificar e identificar trabalhos a fim indicar desafios e dificuldades que fazem parte do processo de formação do professor de Computação. $\mathrm{O}$ fato de identificarmos apenas 50 artigos científicos, em uma pesquisa com estudos dos últimos 20 anos, demonstra que este é um tema pouco estudado e, consequentemente, com pouco aprofundamento teórico e cheio de indagações.

\section{Agradecimentos}

Os autores agradecem à Pró-Reitoria de Ações Afirmativas e Assistência Estudantil (PROAE) da Universidade Federal da Bahia (UFBA) pelo apoio financeiro a esta pesquisa, por meio do Programa SANKOFA (Edital PROAE 15/2016) e ao Grupo de Pesquisa e Extensão em Informática, Educação e Sociedade - Onda Digital (UFBA).

\section{Referências}

Araujo A. L.; Andrade W.; Guerrero D. (2016) Um Mapeamento Sistemático sobre a

\footnotetext{
${ }^{13}$ http://sbc.org.br/files/ComputacaoEducacaoBasica-versaofinal-julho2017.pdf
} 
VI Congresso Brasileiro de Informática na Educação (CBIE 2017)

Anais dos Workshops do VI Congresso Brasileiro de Informática na Educação (WCBIE 2017)

Avaliação do Pensamento Computacional no Brasil. In: Anais dos Workshops do Congresso Brasileiro de Informática na Educação. Vol 5.

França R.; Tedesco P. (2015) Desafios e oportunidades ao ensino do pensamento computacional na educação básica no Brasil. In: Anais dos Workshops do Congresso Brasileiro de Informática na Educação. Vol 4.

Matos, E.; Silva, G. F. B. (2012) Currículo de licenciatura em computação: uma reflexão sobre perfil de formação à luz dos referenciais curriculares da SBC. XX Workshop em Educação em Computação (WEI). In: Anais do XXXII Congresso da Sociedade Brasileira de Computação. s/p.

Matos, E.S. (2013). Identidade profissional docente e o papel da interdisciplinaridade no currículo de licenciatura em computação. Revista Espaço Acadêmico, v. 13, n. 148, p. 26-34, 2013.

. (2017). O ensino de Computação como mecanismo de integração digital.

Computação Brasil, Porto Alegre/RS, p. 48-53, jul/2017.

Pinto, A. V. (2005). O Conceito de Tecnologia. Rio de Janeiro: Contraponto, vol. 2.

Souza, O. S.; Morais, P. S.; Júnior, F. C. S. (2015) Um Estudo sobre a Evasão no Curso de Licenciatura em Informática do IFRN - Campus Natal - Zona Norte. In: XXIII Workshop sobre Educação em Computação (WEI).

Wing, J. M.; Stanzione, D. (2016) Progress in computational thinking, and expanding the HPC community. Communications of the ACM, v. 59, n. 7, p. 10-11. 